\title{
Transmission protocols for challenging networks: LTP and LTP-T
}

\author{
Fahad SYED MUHAMMAD \\ Communication, Propagation and Radar \\ IETR \\ National Institute of Applied Sciences, Rennes \\ France \\ Fahad.Syed-Muhammad@ens.insa-rennes.fr
}

\author{
Laurent FRANCK \\ Microwaves Department \\ ENST Bretagne - Toulouse Site \\ France \\ Laurent.Franck@enst-bretagne.fr
}

\author{
Stephen FARRELL \\ Distributed Systems Group \\ Department of Computer Science \\ Trinity College Dublin \\ Ireland \\ Stephen.Farrell@cs.tcd.ie
}

\begin{abstract}
Challenging networks require transmission protocols able to deal with the specificities of the environment. These adverse conditions include very long delays (delays in hours are normal), episodic connectivity, asymmetric data rates, higher bit error rate (The raw BERs are in the order of $10^{-1}$ ), very high free space losses (around 250-300 dB) and power constraints that may severely compromise the effectiveness of standard transport protocols. Transmissions protocols must also relieve applications and other upper-layer protocols (like the bundle protocol from the Delay Tolerant Network architecture) from the need to deal with the characteristics of intervening communications networks and services. In this paper we analyse the impacts of parameters such as loss probability and propagation delay on Licklider Transmission Protocol (LTP) [1], [2] and the newly proposed Licklider Transmission Protocol-Transport (LTP-T) [3] following a comparative study of both the protocols.
\end{abstract}

\section{INTRODUCTION}

Communication on networks can be challenging specially if they are suffering from high delays and disruptions. In such scenarios the Internet protocol suite is ineffective. A new architecture is required. Interplanetary communications provide the canonical example of severely impacted data communications:

Very long delays Interplanetary networks suffer very long propagation delays. Propagation delay is approximately 4 minutes when Earth and Mars are at their closest approach and can exceed up to 20 minutes when Earth and Mars are in opposition.

Bit error rate In fiber optic systems, bit error rate is as low as $10^{-12}$ to $10^{-15}$, while deep space missions typically operate with BER on the order of $10^{-1}$.

Very high free space losses The losses associated with free space depend on the distance between the two planets which are very large. For example free space losses between Earth are Mars are in the range of 250-300 dB.

Episodic connectivity Because end-to-end connectivity may not be permanent, maintaining a communication path between local and remote endpoints is one of the biggest challenge in such networks.

Asymmetric data rates Asymmetric data rates mean that a system may have a different data rate for outbound traffic than for inbound traffic. It exists in some networks like
ADSL but it is very limited approximately 100:1. In a deep space environment it may be in the order of 1000:1 or higher. The quality of a space link is affected by the sender's power generation capability, the receiver's power amplification capability, the distance between sender and receiver, and the path condition. The link quality is therefore different in opposite directions. The time-dependent nature of the network topology also causes the deep-space links to be asymmetric in delay and stability. Finally, due to application requirements, forward/reverse channels of deep-space communication links have bandwidth asymmetry.

Power constraints The operation of the space elements mainly depends on re-chargeable battery using solar energy. Therefore, routing protocols need to be power efficient.

Special protocols are needed for such type of networks rather than standard Internet protocols. For example, the highly conversational characteristics of TCP coupled to the requirements of end-to-end connectivity makes it unusable. LTP is one of those protocols which are purposely developed for these types of networks from the scratch. LTP-T is a multi-hop, transport extension of LTP.

Licklider Transmission Protocol (LTP) [1], [2] is a transmission protocol for delay-tolerant yet reliable communication between two points and is designed to serve as a convergence-layer protocol for the interplanetary leg(s) of an end-to-end path in a delay-tolerant network. LTP runs just above link layer and may also be useful for some kinds of terrestrial applications (eg sensor networks).

Licklider Transmission Protocol-Transport (LTP-T) [3] is a newly proposed protocol which extends LTP as a transport protocol. It explains the reasons of using transport layer approach on the believe that most delay tolerant networks will be connected to the terrestrial Internet, at some point, so it will be beneficial to use standard naming and addressing scheme at the application layer. Also programmers are familiar with developing applications based on transport layer, so it will be easier to develop and deploy applications. LTP-T is designed for multi-hop environment, so reliability and related issues 
become more complex as we compare it with LTP.

\section{LTP VS LTP-T}

In this section, firstly both the protocol are described and then a rationale of their comparison is given.

\section{A. Licklider Transmission Protocol (LTP)}

The LTP protocol is primarily designed to be run over single links with extremely high latency. The design of LTP differs from that of TCP in several significant ways. Firstly because of several severe inherent constraints on challenging networks discussed above, the assumption of copious computational resources to LTP engines compared to the communication resources available on the link between them is generally taken. Secondly, establishing a communication link across such long distances requires many hardware configuration measures, such as orienting a directional antenna correctly, tuning a transponder to pre-selected transmission and/or reception frequencies and diverting precious electrical power to the transponder at the last possible moment, and for the minimum necessary length of time. It is therefore generally assumed that LTP operating environment must be able to pass information on the link status (termed as "link state cues") to LTP, providing the information about the remote LTP engine(s) currently transmitting to the local LTP engine and vice versa. The operating environment itself must have this information in order to configure communication link hardware correctly.

LTP handles the transmission of a block of data, which may be split into segments that match the maximum transmission unit for the link in question. All of the segments are part of the same unidirectional LTP session. LTP is assumed to be layered over some link layer where the LTP engine is somehow given "cues" as to when it can communicate to a given peer.

LTP operation is divided mainly into red part (reliable transmission) and green part (unreliable transmission) segments. It starts with red part segment transmission and one may include any number (application dependent) of checkpoints. A special red part segment, the EORP (End Of Red Part), must be a checkpoint. On the reception of a checkpoint, report segments, containing report claims, are sent to the transmitter which are acknowledged by report acknowledgment segments. At the reception of an EOB (End Of Block), the entire data is delivered to application layer. A sample time sequence diagram of LTP is shown in Figure 1. Multiple transmission between two peers may be in progress concurrently.

LTP tolerates link interruptions without data loss and is designed to impose minimal overhead on low-capacity and/or asymmetric links. Segments can be extended (e.g., security coding). Transmission or reception of individual blocks may be canceled while accelerated retransmission, i.e. multiple

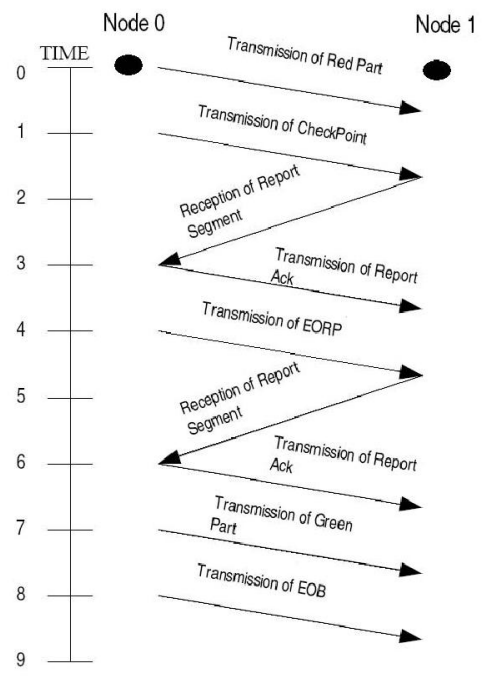

Fig. 1. Time sequence diagram of LTP

checkpoints per transmitted block, is also possible. LTP can provide both reliable and unreliable functionality concurrently on a single link.

\section{B. Licklider Transmission Protocol-Transport (LTP-T)}

LTP-T is designed for multi-hop environments, so reliability and related issues become more complex compared to LTP. When no error occurs, LTP-T basically behaves like a sequence of independent LTP sessions (one for each hop). On the other hand, when there is segment loss or corruption, successfully received segments are forwarded to the next node while error recovery is initiated for the other ones. This leads to a de-synchronisation of the initial segment sequence and calls for proper checkpoint re-scheduling. LTP-T is work in progress and while mechanisms like congestion control and checkpoint scheduling have been identified, their actual performance evaluation.

\section{Why comparing the two protocols?}

These two protocols are very different in their approach i.e. at one side LTP follows a point-to-point approach while on the other, LTP-T proposes a multi-hop approach. This contribution addresses the following issues:

- What gain/losses are expected from splitting a communication path featuring challenging conditions into many hops ?

- In LTP and LTP-T, what are the tradeoffs driving reliability, goodput and end-to-end delay.

These aspects are investigated in several environments (deep space and sensor networks) for applications displaying different traffic characteristics and service requirements (image, movie and bulk data transfer). The performance metrics used are the goodput and the end-to-end delay. 


\begin{tabular}{|c|c|c|c|}
\hline Scenario & $\begin{array}{l}\text { Number } \\
\text { of nodes }\end{array}$ & $\begin{array}{l}\text { Propagation } \\
\text { delays (TU) }\end{array}$ & $\begin{array}{c}\text { Frame } \\
\text { loss ratio }\end{array}$ \\
\hline Deep space & 4 & $\{600000,10,10\}$ & $\begin{array}{c}\left\{10^{-2}, 10^{-6}\right. \\
\left., 10^{-6}\right\} \text { or } \\
\left\{10^{-1}, 10^{-6}\right. \\
\left.10^{-6}\right\}\end{array}$ \\
\hline Sensor net. & 11 & $\begin{array}{c}10^{*} 50 \mathrm{TU} \text { or } \\
10^{*} 100 \mathrm{TU} \text { or } \\
10 * 200 \mathrm{TU}\end{array}$ & $10^{*} 10^{-2}$ \\
\hline
\end{tabular}

TABLE I

NETWORK CHARACTERISTICS: DEEP SPACE DISPLAYS DIFFERENT FR AME LOSS RATIOS AND FIXED PROPAGATION DELAYS. SENSOR NETWORK USES DIFFERENT PROPAGATION DELAYS AND FIXED FRAME LOSS RATIOS.

\section{Simulation RESUlts}

\section{A. Simulation environment}

The simulations consider the operation of LTP-T over two types of networks: deep space and sensor network. The deep space network displays a propagation delay and frame loss ratio (FLR) asymmetry between the first hop and the two other hops as it happens in a deep space - lander rover communication chain. The sensor network features 10 hops with equal and less challenging propagation delays and FLRs. Table I summarizes the parameters of these networks. Delays are expressed in TU (time unit). LTP being a single hop protocol, equivalent one-hop environments are derived consisting of the summed propagation delays and frame loss ratios (LTP-T assumes independent uniform error distributions at each hop).

Three applications are evaluated: image, movie and bulk data transfers. These applications differ from the number of red segments out of the 4000 transmitted segments. For image transmission, only the header of the image is protected (i.e. tagged as red). For movie transmission, intra frames are protected while predicted frames are not. Finally, for bulk data transmission $90 \%$ of data frames are protected. Whatever the application, transmitting one segment eats 1 TU plus the propagation delay as indicated before. These parameters are summarised in Table II.

\begin{tabular}{|c|c|c|}
\hline Application & $\begin{array}{c}\text { Number of } \\
\text { red segments }\end{array}$ & $\begin{array}{c}\text { Number of } \\
\text { green segments }\end{array}$ \\
\hline Image & 200 & 3800 \\
\hline Move & 600 & 3400 \\
\hline Bulk data & 3600 & 400 \\
\hline
\end{tabular}

TABLE II

APPLICATION CHARACTERISTICS

Finally, each (environment,application) couple is tested against different checkpoint ratios, respectively $5 \%, 10 \%$, $20 \%$ and $50 \%$ of the number of red segments. Checkpoints are equally distributed. Two performance metrics are considered: the end-to-end delay and the goodput. The end-to-end delay denotes the time required to transfer all red and green segments (taking into account that some green segments may be lost). The goodput denotes the transmission effort required for sending all segments and is expressed as the ratio between the number of red segments to the application has to send and the number of red segments actually transmitted.

The simulator is of discreet event type written in C language. Every single scenario is run 100 times and the results (end-to-end delay and goodput) are averaged.

\section{B. Performance of LTP-T in a deep space scenario}

Figure 2 shows the end-to-end delay as a function of the checkpoint ratio for different applications and FLRs. In a deep space environment, a high number of red parts and frame losses yield an increased end-to-end delay. Indeed, these two parameters may cause more retransmissions. Increasing the checkpoint ratio also yields a higher end-to-end delay. This is due to the long duration of the control loop (i.e. sending data and receiving report segments) between the first and second node: if the number of segments in the loop is low (as it is the case for large checkpoint ratios), recovering from several errors takes more time. In an actual implementation, the duration of control loops should be kept as low as possible by prioritizing report segments on the return links. Networks with low propagation delays (hence short control loops) are not affected as it will be shown later.

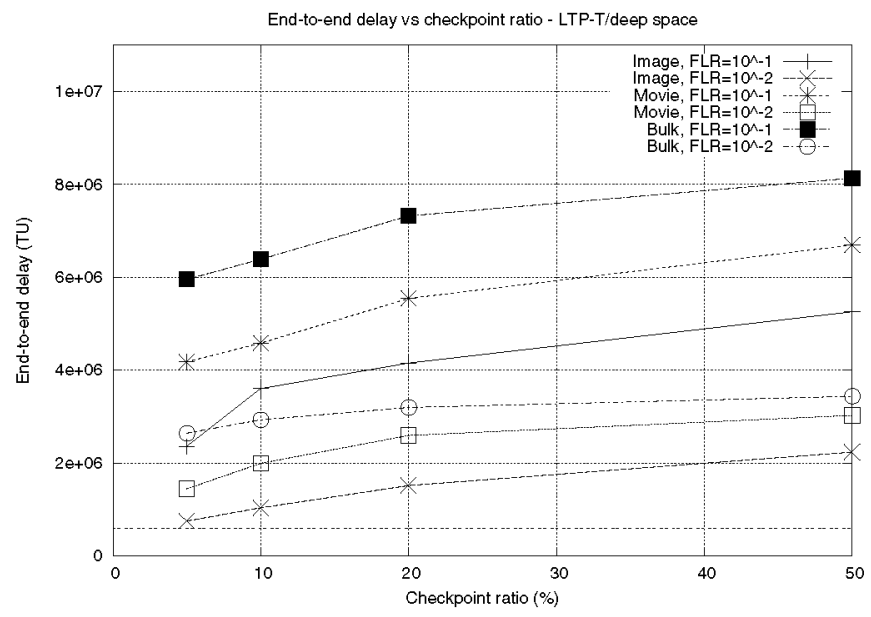

Fig. 2. End-to-end delay (TU) as a function of the checkpoint ratio in a deep space/LTP-T environment. Image, movie and bulk data transfer are represented for different frame loss ratios. The bottom line shows the cumulated propagation delays for the 3 hops (600020 TU).

Figure 3 shows the goodput considering the same parameters as in Figure 2. The curves are overlaid for equal frame loss ratios. When losses are important $\left(10^{-1}\right)$, increasing the checkpoint ratio slightly improves the goodput upon two occasions: loss of a report segment and loss of a red segment with checkpoint. In these two cases, the source must retransmit all data segments within the checkpoint scope. Narrowing the 
checkpoint scope contributes to impair the cost of retransmission (this also holds for LTP). Implementations should also take care to increase coding robustness for segments containing checkpoints and reports.

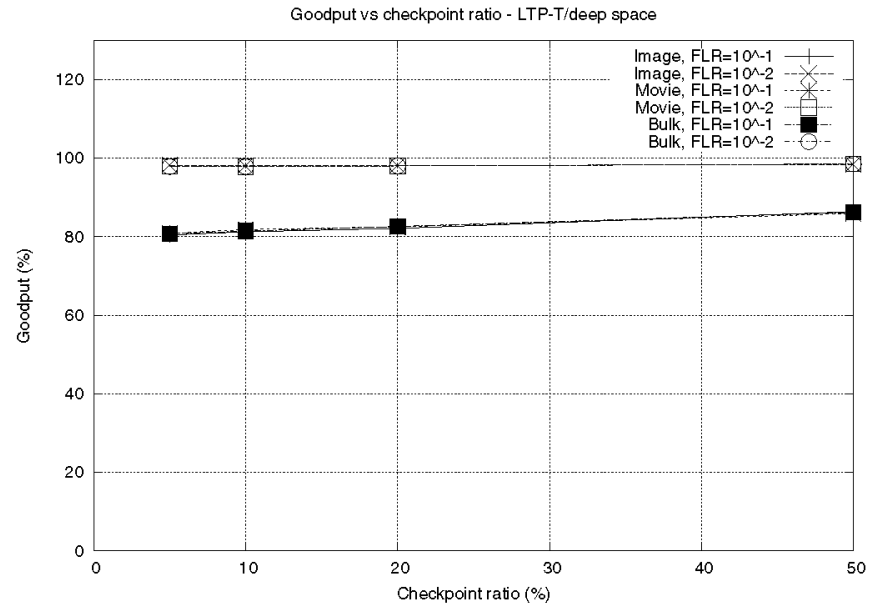

Fig. 3. Goodput $(\%)$ as a function of the checkpoint ratio in a deep space/LTP-T environment. Image, movie and bulk data transfer are represented for different frame loss ratios. Curves are overlaid for equal frame loss ratios.

\section{Performance of LTP-T in a sensor network scenario}

Figure 4 shows the end-to-end delay as a function of the checkpoint ratio for different applications and propagation delays. For a propagation delay of $50 \mathrm{TU}$ per hop, the three applications present similar end-to-end delays. When the propagation delay increases to $100 \mathrm{TU}$, a small difference starts to appear between bulk data and the applications with fewer red parts (image and movie). This trend is confirmed when the propagation delay is set to $200 \mathrm{TU}$. When propagation delay is low (50 TU), error recovery is fast, not imparing the end-to-end delay. Also, with a frame loss ratio of $10^{-2}$, retransmissions are seldom required especially for those applications where red segments are not the majority.

Additionally, the curves appear to be almost insensitive to the checkpoint ratio except for cases where there is a high number of red parts and a large propagation delay. These effects were already witnessed for the deep space scenario where larger delays increase the duration of the control loops hence the end-to-end delay.

Figure 5 shows the goodput considering the same parameters as in Figure 4. All curves are overlaid since the frame loss ratio is equal. For the same reason as described in the deep space scenario, increasing the checkpoint ratio improves goodput. However, contrary to the deep space scenario, goodput improvement is not achieved at the expense of a large end-to-end delay.

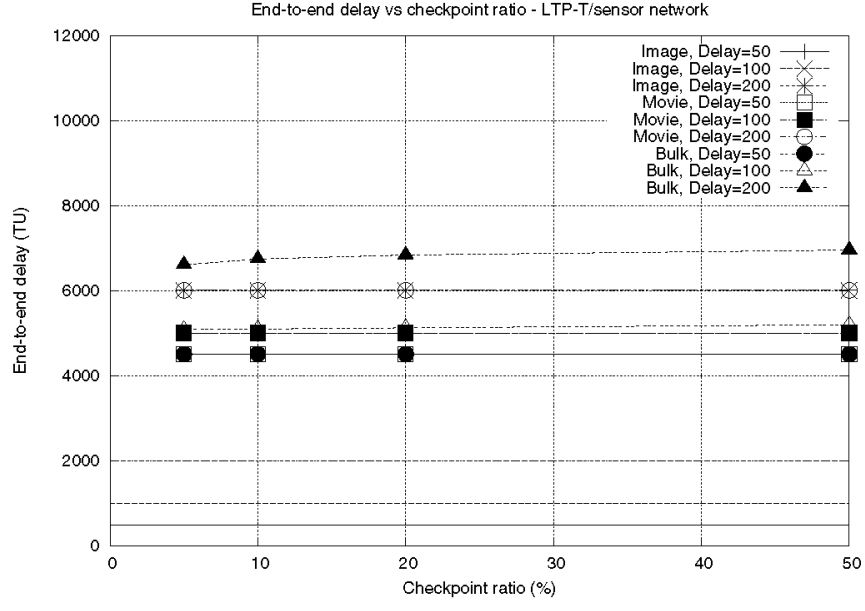

Fig. 4. End-to-end delay (TU) as a function of the checkpoint ratio in sensor network/LTP-T environment. Image, movie and bulk data transfer are represented for different propagation delays. For a propagation delay of 50 TU, the image, movie and bulk data curves are overlaid. The same goes for the image and movie curves when propagation delays are equal to 100 and 200 TU. The three bottom lines show the cumulated propagation delays for the 10 hops $(500,1000$ and $2000 \mathrm{TU})$.

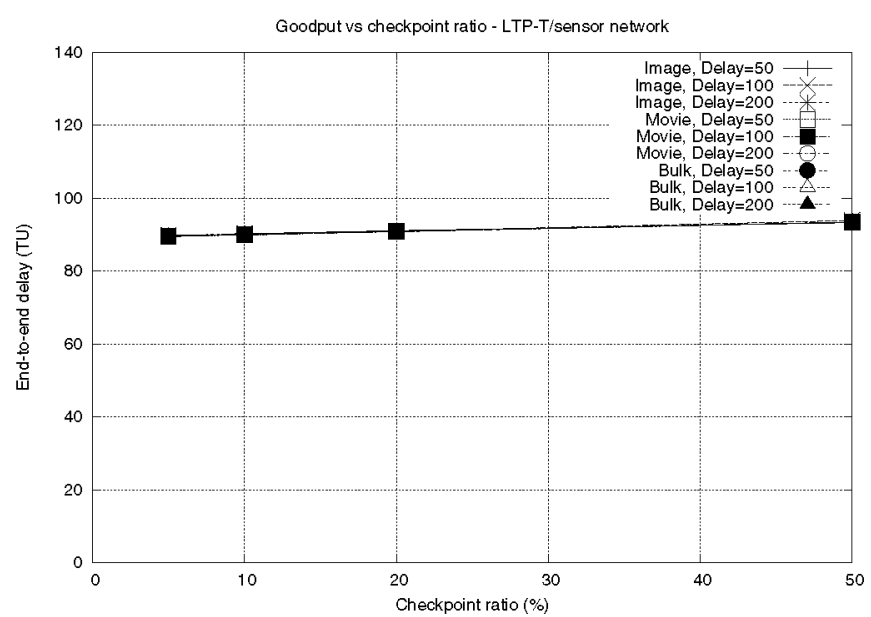

Fig. 5. Goodput $(\%)$ as a function of the checkpoint ratio in a sensor network/LTP-T environment. Image, movie and bulk data transfer are represented for different propagation delays. All curves are overlaid.

\section{Single (LTP) vs Multi-hop (LTP-T) approaches}

This section compares the performance of LTP-T and LTP for deep space and sensor networks. From this comparison, one can derive the benefits of using a multi-hop approach whenever possible.

Table III shows how LTP and LTP-T perform in a deep space environment considering a frame loss ratio of $10^{-1}$ and a checkpoint ratio of $20 \%$. Both LTP and LTP-T display comparable end-to-end delays. Indeed, in the deep space scenario the duration of the second and third hop are negligible compared the first hop. Treating the whole path as a single (LTP) hop makes no difference as far as the end-to-end 
delay is concerned. On the other hand, LTP-T displays a goodput performance about $10 \%$ larger than LTP. The multi-hop approach makes it possible to isolate a challenging first hop as far as goodput is concerned. Retransmissions occurring on the second and third hops are quickly resolved as opposed to the single hop LTP.

\begin{tabular}{|c|c|c|c|}
\hline \multicolumn{4}{|c|}{ Deep space (FLR $=10^{-1}, \mathrm{CP}$ Ratio=20\%) } \\
\hline Protocol & Image & Movie & Bulk \\
\hline LTP-T & $4152157 \mathrm{TU}$ & $5544523 \mathrm{TU}$ & $7322198 \mathrm{TU}$ \\
& $82.11 \%$ & $82.51 \%$ & $82.60 \%$ \\
\hline LTP & $4368291 \mathrm{TU}$ & $5928742 \mathrm{TU}$ & $7166674 \mathrm{TU}$ \\
& $71.11 \%$ & $71.11 \%$ & $71.45 \%$ \\
\hline
\end{tabular}

TABLE III

END-TO-END DELAY (TU) AND GOODPUT (\%) FOR LTP-T AND LTP IN A DEEP SPACE SCENARIO.

Table III shows how LTP and LTP-T perform in a sensor network environment considering a frame loss ratio of $10^{-2}$ for each LTP-T hop, a checkpoint ratio of $20 \%$ and a propagation delay of 100 TU. A multi-hop (LTP-T) approach outperforms in all cases a single hop approach (similar conclusions are made for propagation delays of 50 and 200 TU). As indicated before, keeping control loops short (100 TU instead of 1000 TU in this case) speeds up reactions on transmission errors especially for applications displaying a large number of red parts (like bulk data transfer).

\begin{tabular}{|c|c|c|c|}
\hline \multicolumn{4}{|c|}{ Sensor network (Prop. delay=100, CP Ratio=20\%) } \\
\hline Protocol & Image & Movie & Bulk \\
\hline LTP-T & $5009 \mathrm{TU}$ & $5009 \mathrm{TU}$ & $5130 \mathrm{TU}$ \\
& $90.89 \%$ & $90.92 \%$ & $90.84 \%$ \\
\hline LTP & $7716 \mathrm{TU}$ & $10121 \mathrm{TU}$ & $14617 \mathrm{TU}$ \\
& $71.05 \%$ & $71.49 \%$ & $71.34 \%$ \\
\hline
\end{tabular}

TABLE IV

END-TO-END DELAY (TU) AND GOODPUT (\%) FOR LTP-T AND LTP IN A SENSOR NETWORK SCENARIO

\section{CONCLUSION}

This paper presented two approaches for transmitting data in challenging environments like deep space and low power sensor networks. LTP is a single hop transmission protocol designed for large propagation delays and frame loss ratios. LTP makes use of partial reliability in order to minimise interactions between endpoints. LTP-T is the multi-hop extension and more or less consists in a sequence of LTP sessions.

The performance of LTP-T is evaluated for the two environments considering applications (image, movie and bulk data transfers) with different requirements. The endto-end delay and goodput serve as performance metrics. Several effects are identified closely related to the processing of retransmissions in case of errors (missing or corrupted segments).

Then, single and multi-hop approaches (LTP and LTP-T respectively) are compared. It is shown that in the example scenarios, that deploying a multi-hop approach contributes to the control of goodput and end-to-end delay, either by keeping control loops efficient or by isolating hops that are challenging (from a propagation delay or a frame loss ratio standpoint).

Future work includes a close investigation of LTP-T specific mechanisms like congestion control and checkpoint scheduling among subsequent hops.

\section{REFERENCES}

[1] Ramadas, M., Burleigh, S., and Farrell, S., "Licklider Transmission Protocol - Specification", Internet Draft. April 2007.

[2] Burleigh, S., Ramadas, M., and Farrell, S., "Licklider Transmission Motivation", Internet Draft. April 2007.

[3] Farrell, S., Cahill, V., "LTP-T: A Generic Delay Tolerant Transport Protocol", Technical Report, Trinity College Dublin, 2005.

[4] "TM Synchronization and Channel Coding.", CCSDS Blue Book. Issue 1. September 2003. 